\title{
A Force/Position Hybrid Controller for Rehabilitation Robot
}

\author{
J. Hua, L.L. He, Z.Q. Kang, K.D. Yan
}

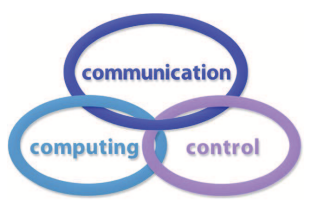

\author{
Jin Hua* \\ Xi'an University of Architecture and Technology, \\ School of Mechanical and Electrical Engineering \\ Xian 710055, China \\ Xi'an Technological University, \\ College of Electronic Information Engineering \\ Xi'an 710021, China \\ *Corresponding author: huahua_dz@aliyun.com
}

\section{Lile $\mathbf{H e}$}

Xi'an University of Architecture and Technology,

School of Mechanical and Electrical Engineering

Xi'an 710055, China

\section{Zhiqiang Kang}

Xi'an University of Architecture and Technology,

School of Mechanical and Electrical Engineering

Xi'an 710055, China

\section{Keding Yan}

Xi'an Technological University,

College of Electronic Information Engineering

Xi'an710021, China

\begin{abstract}
The growing ageing population in China poses a huge demand for rehabilitation care, which can be satisfied by the latest robot technology. Focusing on the motion system of a six degrees-of-freedom (DOF) robot, this paper explores the relationship between the force, torque, velocity and the postures of the end effector and joint. Drawing on robot control theories, the existing manipulator force/position hybrid controllers were reviewed, and a force/position hybrid controller was designed for path planning of rehabilitation robot. Then, the robot was modelled on the Robot Operating System (ROS), using the Unified Robot Description Format (URDF) file and the MoveIt! Setup Assistant. Finally, our controller was tested in the ROS virtual simulation environment. The results show that our controller can facilitate and optimize the design of the path of rehabilitation robot.

Keywords: rehabilitation robot, compliance control, Bezier curve, robot operating system (ROS).
\end{abstract}

\section{Introduction}

The ageing problem has become increasingly serious in China since the 1990s. From 1990 to 2014, the elderly population, defined as people aged 65 and over, increased from 62.99 million $(5.57 \%)$ to 88.11 million (6.96\%). The elderly population is expected to account for over $20 \%$ 
in the total population of China by 2040. Currently, tens of millions of elderlies in China need rehabilitation care, posing a huge pressure on the medical and insurance systems.

Many industrial robots have been modified to provide rehabilitation care to the elderlies. For example, Li and Ye [8] created a motion relationship mapping model for upper limb joints, established several rehabilitation robots based on the relationship, and used joint torque signals to control the motion of the robots. Wang [18] developed a highly flexible pneumatic upper limb rehabilitation robot, which effectively prevents secondary damage to the affected limb. Pan and Song [12] designed an upper limb rehabilitation robot using impedance control strategy.

The existing rehabilitation robots mainly adopt the structure of a multi-degrees of freedom (DOF) exoskeleton with a single manipulator. The motions of the manipulator are controlled based on the signals of muscle force on the affected limb. This control mode cannot obtain the user's movement intention easily, or achieve good real-time response and accuracy.

Drawing on robot control theories, the existing manipulator force/position hybrid controllers were reviewed, and a force/position hybrid controller was designed for path planning of rehabilitation robot. Then, the robot was modelled on the Robot Operating System (ROS), using the Unified Robot Description Format (URDF) file and the MoveIt! Setup Assistant. Finally, our controller was tested in the ROS virtual simulation environment. The results show that our controller can facilitate and optimize the design of the path of rehabilitation robot.

\section{Literature review}

Robot technology is highly interdisciplinary and integrated. It covers many different fields, such as automatic control, computer technology, advanced sensing and mechanism. In recent years, more and more robots have been used to provide special services.

The compliance control is the key and difficult point in the research of robot technology. The compliance means the manipulator can adapt to the changes in the environment, keeping its contact force with the environment at a desired level. Over the years, many methods have been developed for compliance control of robot, including impedance control (converting feedback force into position or speed for dynamic adjustment), force/position mixed control, stiffness control [15], and implicit force control [9]. Being the hybrid of force control and position control, the compliance control can be achieved either actively or passively.

Under active compliance control, the robot perceives and regulates the contact force, completing the mixed control of its force and position. Considering the uncertainty of contact stiffness, Hogan [3] designs a force feedforward controller by inverse dynamics approach, and realizes parallel control of robot force and position. Sun and Chen [17] proposes a robot control method coupling motion prediction and impedance control, which ensures that the manipulator is flexible to react with unknown restricted mechanisms. Xu [22] puts forward a discrete-time sliding mode impedance controller with adaptive gain, and applies the controller to accurately control the force and position of a micro-gripper using piezoelectric bimorphs under uncertainties and interferences. Based on force/position hybrid control, Chang et al. [2] develops a fuzzy control method for redundant manipulator, which guarantees the accuracy of the end effector without sacrificing autonomy and flexibility. Singh and Sukavanam [16] creates a robust adaptive force/position hybrid control strategy based on neural network (NN). Under this strategy, the robot dynamics with uncertainties and disturbances are described by feedforward neural network (FNN), laying the basis for effective control of the force and position of the end effector. Hu et al. [4] conducts orthogonal separation of force control and position control in the time domain, and realizes grinding and polishing of the aspherical surface through mixed control of both force and position. 
Under passive compliance control, the robot position is controlled by the rigid robot, while the contact force is controlled by a flexible joint added to the end of the robot. Compared with the active mode, passive compliance control is easy to implement, rich in economic benefits and widely applicable in industries. Nonetheless, the high demand on precision limits the use of passive compliance control in fast-responding applications. The most famous passive compliance controller is the remote center compliance (RCC) device developed by Whitney and Rourke [21]. The RCC device has been adopted for assembly operations to achieve compliance with any compliance center. However, this device cannot adapt well to operations with different stiffness requirements, due to its inflexible compliance center and fixed stiffness. Beijing University of Technology [14] designed a pneumatically-driven passive-compliance robotic hand that can control the contract force at a low level and complete precision hole and shaft assemblage. The robotic hand consists of an air bag and an air bearing. The former reduces the angular stiffness and the latter lowers the lateral stiffness. Harbin Engineering University [20] presented a passive-compliance flexible gripper for underwater search and object grabbing. The gripper has two fingers, a removable wrist, easily replaceable end effectors, a support mechanism, an active locking mechanism and a passive compliance mechanism. Despite the lack of a single DOF, the gripper can grab objects in different postures. Huang et al. [5] introduces a compliance mechanism to the abrasive belt of a robotic polishing system. The mechanism can adapt to the changing contact force of the system, eliminating the need of manual grinding of turbine blades. To cope with dynamic changes of the working environment and the uncertainties of human-computer interaction (HCI), Zhang et al. [23] designs a manipulator with passive compliance structure and active compliance control, and adds several elastic drive modules between the joint motor and connecting rod. Baksys et al. [1] set up an electric vibration table with a compliance device, examines the stress of the vibration table, and discloses the relationship between vibration frequency and assembly time. Kilikevicius and Baksys [7] analyzes the effects of dynamic systems and excitation parameters on passive compliance assembly by vibrating the casing in the axial direction on the platform basis. Huang et al. [6] puts forward a passive compliance control strategy for the position and force of a robotic polishing system, and installs a compliance device at the end effector for force control.

From the above, Because of the manipulator dynamics model contains the uncertainty structures and parameters, even more, the external disturbance, the method based on the model control will be resulted in the system control quality decline, more over instability from different degrees affection. Therefore, the adaptive control method which is selected in this paper, is proposed to deal with these uncertainties and external disturbances. The application of compliant control technology in service robot becomes hotter, but more difficult in the process of interacting with human. In this work, the force/position hybrid control method is adopted to solve the operation task of assisted rehabilitation movement. Combined with self-adaptation control, it structured the model which is contains the robot manipulator and the contact environment as a whole.

\section{Design of force/position control algorithm for robotic manipu- lator}

Mechanical impedance is the ratio of the increment of dynamic force in any DOF and the resulting increment of dynamic displacement. This ratio is generally a nonlinear coefficient. Therefore, when the manipulator is in contact with the working environment, both the position of the end effector and the dynamic relationship between the position and the contact force should be controlled to adjust the input torque of the manipulator.Currently, the two control 
tasks are mainly implemented by two methods. The first method is the transform of the force signal into position or velocity, followed by the dynamic adjustment of the stiffness and damping part. The second method is the hybrid control of force/position.

The hybrid position/force control decomposes the task space into two orthogonal subspaces for position control and force control. The position is controlled in the tangent subspace under environmental constraints, while the force is controlled in the normal subspace under environmental constraints. In this way, the position/force control is decoupled.

Let $K$ and $P$ be the kinetic energy and potential energy of the manipulator, respectively. Then, the Lagrange equation of the manipulator can be expressed as $L=K-P$. Thus, the following equation can be derived:

$$
\tau=\frac{d}{d t} \frac{d L}{d \dot{q}}-\frac{d L}{d q}
$$

Therefore, the dynamic equation of the manipulator can be defined as:

$$
\tau=M_{i}\left(q_{i}\right) \ddot{q}_{i}+h_{i}\left(q_{i}, \dot{q}_{i}\right)+G_{i}\left(q_{i}\right)
$$

where $M(q), h(q, \dot{q}), G(q)$ are the inertial matrix, centrifugal force and gravity vector of the manipulator, respectively. In the force space, the force acting on the end effector can be mapped to the equivalent joint space moment by the transposition $J^{T}$ of the Jacobian matrix of the manipulator. When the end of the manipulator contacted with the external environment, there will be an $F_{e}$ interaction between the manipulator and the external environment. Hence, in order to maintain the manipulator balance state, the output of torque controlling is expressed as follows:

$$
\tau=M(q) \ddot{q}+h(q, \dot{q})+G_{q}-J^{T} F_{e}
$$

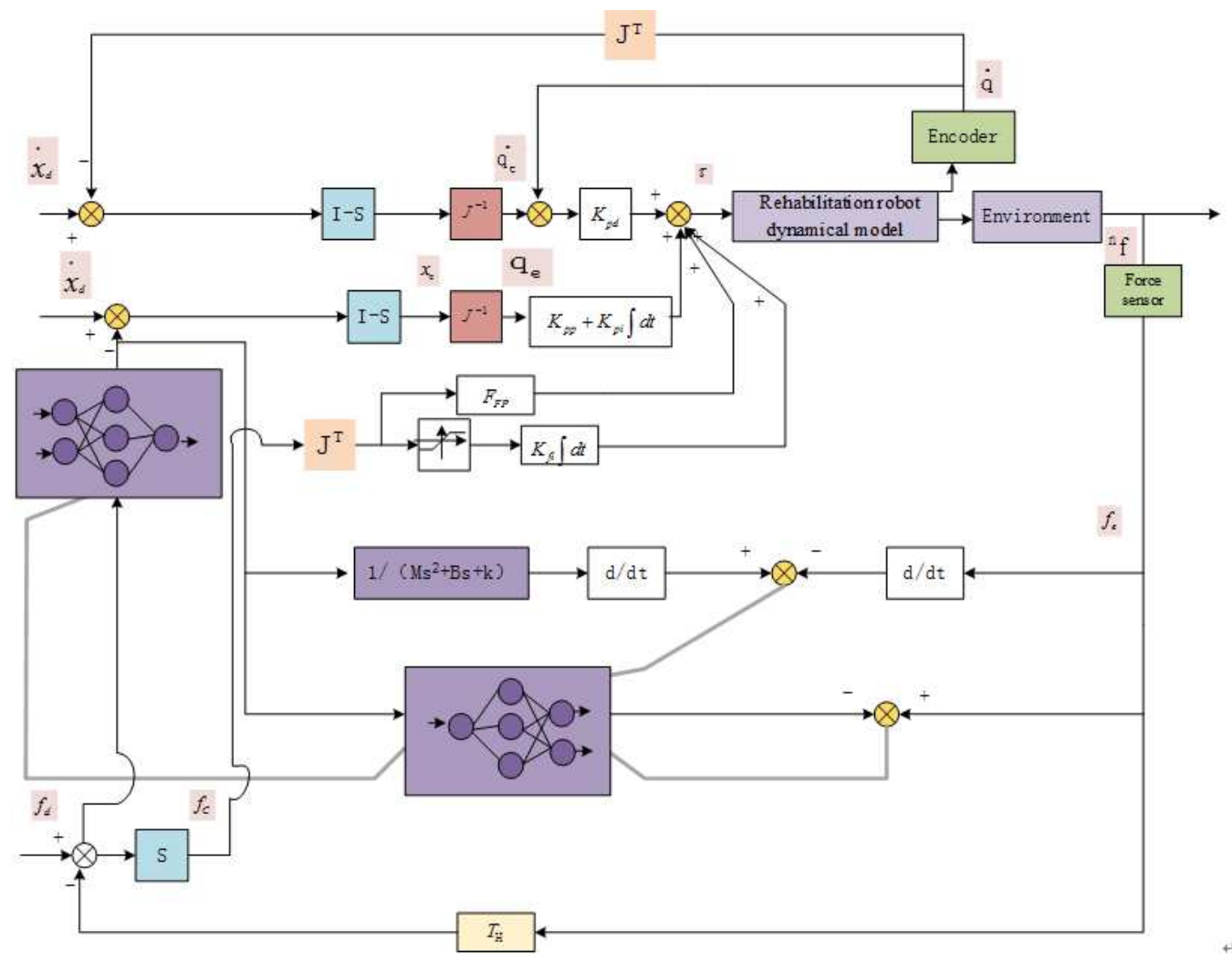

Figure 1: Design of Our Force / Position Hybrid Controller 
The design of our force/position hybrid controller is shown in Figure 1. The output torque of the controller is the sum of the torque computed by the position control information via the inverse Jacobian matrix $J^{-1}$ and that computed by the force control information via $J^{T}$. The inverse Jacobian matrix stands for the mapping relationship between the change of the coordinate velocity of each member in the Cartesian coordinate system and the change of the joint angular velocity of that member.Considering that if only basic impedance control is used to maintain the dynamic relationship between the effector of the robot and the response contact force of the rehabilitation patients' limbs, although smooth contact is achieved to reduce the impact in contact, it cannot achieve good control of the rehabilitation intensity.So the controller is designed by adding artificial neural network, so as to correct the expected output impact using the error between the output of the reference model and the actual output.

In the traditional force/position control algorithm, the adaptive part of environmental parameters is improved. The contact force at the robot effector is measured by the six-dimensional force/torque sensor, which is used as the control part of the force feedback to the force/position hybrid controller. After learning the adaptive modified NN, the position will be changed.So, we select the parameter model of the environment as $M_{d} s^{2}+B_{d} s+K$. When, $M_{d}$ is the target inertia of the impedance model, $B_{d}$ is the target damping, and $K_{d}$ is the target stiffness matrix.

In the controller, $S$ is a diagonal matrix. If $i=6$, then the matrix is a unit matrix of $6 * 6$. Therefore, $\bar{S}$ can be obtained as:

$$
\bar{S}=I-S
$$

The essence of the hybrid control is to set the DOF for the desired path of the end effector, that is, to select the control component of the controller through $S$ and $\bar{S}$.

The cartesian force, which on the end of the manipulator, maps the equivalent joint torque according to the transpose Jacobian matrix $J^{T}$. When the manipulator performs the action task, it is inevitable that it will have physical contact with the external environment or the operating object. As the task executing, manipulator will inevitably contact with the external environment or the operating object. That leads to the results of positional constraints in the positional space.

In addition to considering the dynamic model of the robot arm itself, the model of the interaction between the robot arm and the external environment should be established when modeling the control object, so as to realize the flexible control of the task action process by the dynamic model. Considering the dynamic model of the arm, while the model of the interaction between the robot and environment will be confirmed, so as to achieve the flexible control process by the whole dynamic model. The force sensor is mounted between the end gripper and the joint arm. The data, which acquired from the experiment in a certain rehabilitation teaching movement, are the position and the six-axis force obtain from the force sensor. From that measurement data, the interaction force of this kind rehabilitation exercise between the manipulator and the environment is obtained. In the diagram, the $\tau$ is the total driving torque of the six joints $(i=6)$. The joint position q and joint velocity can be measured by the photoelectric encoder. Acquired by six-axis torque sensor, ${ }^{H_{\mathrm{f}}}$ is converted to the effective vector ${ }^{c} \mathrm{f}$ in cartesian coordinate system by using the coordinate transformation.

So suppose this is a S selection matrix, which elements in the diagonal matrix are set to 1, corresponding to its degree space are controlled by the applied force loop. For example, when:

$$
S=\operatorname{diag}[1,0,1,0,1,1]
$$

so $X_{d}=\left[x_{d}, 0, z_{d}, 0, \beta_{d}, \gamma_{d}\right]$, As known from the formula, the expected displacement of the Y-axis and the rotation around the $\mathrm{Y}$ axis should be ignored. As the same as in the position control ring. In addition, the original six-degree-of-freedom manipulator become a redundant mechanical manipulator. Meanwhile, the change of the expected force is controlled by the force closed loop. 
The motion differential equation is:

$$
\dot{X}_{e}=J \dot{\Theta}
$$

so as to, $\dot{X}_{e}$ is the $m \times l$ dimensional vector, that is composed of linear velocity and angular velocity of the end effector, $\dot{\Theta}$ is the $n \times l$ dimensional vector, composed of angular velocity of each joint in manipulator, while, $J$ is $m \times n$ matrix. When $J$ is singular matrix, that means $m<n$, the joint angular velocity of redundant manipulator is:

$$
\dot{\Theta}=J^{+} \dot{X} e+\left(I-J^{+} J\right) \dot{\varphi}
$$

in this formula, $J^{+}$is the pseudo-inverse of $J$, if $J$ is full rank, then $J^{+}=J^{T}\left(J J^{T}\right)^{-1}$. $I$ is the identity matrix, $\left(I-J^{+} J\right)$ is the null space projection matrix, and is any joint angular velocity vector. When $\dot{\phi}=0, \dot{\Theta}$ can be solved with the minimum norm solution. In other words, the motion of the end effector can be guaranteed under the control of the minimum joint velocity in joint space, or use $K \nabla H(\Theta)$ instead of $\dot{\Theta}$ :

$$
\dot{\Theta}=J^{+} \dot{X}_{e}+k\left(I-J^{+} J\right) \nabla H(\Theta)
$$

where the $H(\Theta)$ can be optimized by the gradient projection method.

\section{Robot trajectory planning based on Bezier curve}

The Bezier curve can be defined as:

$$
b(u)=\sum_{j=0}^{m} B_{j}^{m}(u) p_{j}, \quad 0 \leq u \leq 1
$$

where $p_{j}$ is the control point; $B_{j}^{m}(u)$ is the M-order Bernstein polynomial:

$$
B_{j}^{m}(u)=\frac{m !}{j !(m-j) !} u^{j}(1-u)^{m-j}
$$

The polynomial form of the Bezier curve can be expressed as:

$$
b(u)=\sum_{i=0}^{m} a_{j} u^{i}
$$

where $a_{j}$ is a coefficient determined by control point $P_{j}$ :

$$
a_{j}=\frac{m !}{(m-i) !} \sum_{j=0}^{i} \frac{(-1)^{j+i}}{j !(i-j) !} P_{j}
$$

According to the principle of Bezier curve principle, a planned path for the rehabilitation robot was obtained (Figure 2).

As shown in Figure 4, the planned path consists of a straight segment $Q_{A} Q_{B}$ and an arc segment $Q_{B} Q_{C}$; the origin and radius of the arc segment are $O_{A}$ and $R$, respectively; the fifthorder Bezier transition segment is $Q_{B}^{\prime} Q_{B}^{\prime \prime}$. The straight segment reaches the front of the target slag, which is at the center of the arc segment, while the arc segment bypasses the target slag to complete the slagging action. 


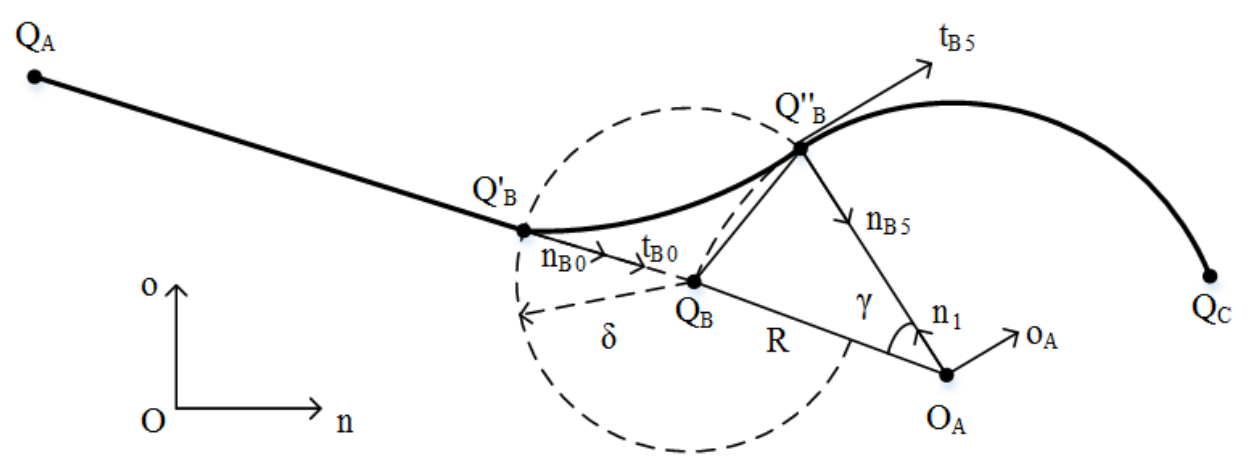

Figure 2: Trajectory Planning for The Rehabilitation Robot

Let $\delta$ be the tolerance of the fifth-order Bezier transition segment. Then, the $Q_{B} Q_{B}^{\prime \prime}$ of center angle $\gamma$ can be obtained by triangulation:

$$
\gamma=2 \arctan (\delta / 2 R)
$$

Let $T$ be the transformation matrix from the global coordinate system $O-Z Y X$ to the arc segment coordinate system $O_{A}-X_{A} Y_{A} Z_{A}$. Then, the two ends of the fifth-order Bezier transition segment $\left(Q_{B_{0}}\right.$ and $\left.Q_{B_{5}}\right)$ can be respectively expressed as:

$$
\begin{gathered}
Q_{B}^{\prime}=Q_{B}-\frac{Q_{B}-Q_{A}}{\left|Q_{B}-Q_{A}\right|} \delta \\
Q_{B}^{\prime \prime}=O+T(R \cos \gamma \quad R \sin \gamma \quad 0)^{T}
\end{gathered}
$$

The six points of the fifth-order Bezier transition segment can be obtained as:

$$
\left\{\begin{array}{l}
Q_{B_{0}}=Q_{B}^{\prime} \\
Q_{B_{1}}=Q_{B_{0}}+\left(\alpha_{B} / 5\right) \cdot t_{B_{0}} \\
Q_{B_{2}}=2 Q_{B_{1}}-Q_{B_{0}} \\
Q_{B_{5}}=Q_{B}^{\prime \prime} \\
Q_{B_{4}}=Q_{B_{5}}-\left(\alpha_{B} / 5\right) \cdot t_{B_{5}} \\
Q_{B_{3}}=2 Q_{B_{4}}-Q_{B_{5}}+\left.0.05\left(\frac{d b}{d u}\right)^{2}\right|_{u=1} \cdot \frac{\vec{R}}{R^{2}}
\end{array}\right.
$$

where $u \in[0,1]$ is the fifth-order displacement parameter of Bezier curve $b=b(u)(u=u(t))$; $\alpha_{B}$ is the first-order differential vector; $\vec{R}$ is the vector pointing from the new start point of the arc segment to the center of the circle. The straight segment and Bezier curve contain the same unit tangential vector at the transition point $t$ and curvature vector $n$ in $Q_{B}^{\prime}$. The unit vector along the direction of $Q_{B}^{\prime} Q_{B}$ can be expressed as:

$$
t_{B_{0}}=n_{B_{0}}=\frac{Q_{B}-Q_{B}^{\prime}}{\delta}
$$

The velocity at the intersection of the arc segment and the Bezier curve is the same as the direction:

$$
t_{B_{5}}=o_{A}
$$

To constraint the start and end of the straight segment, the continuous velocity and acceleration, straight segment displacement, acceleration, maximum velocity, maximum acceleration and 
maximum jerk must satisfy: $u_{0}=0, u_{1}=1, \ddot{u}_{0}=\ddot{u}_{1}=0, \dot{u}_{\max }=v_{\max } / l_{A B}, \ddot{u}_{\max }=a_{\max } / l_{A B}$ and $\ddot{u}_{\max }=j_{\max } / l_{A B}$.

To satisfy the velocity requirement, the straight segment and the fifth-order Bezier transition segment must satisfy:

$$
\left.\frac{d l_{A B}(u)}{d t}\right|_{u=1}=\left.\frac{d b(u)}{d t}\right|_{u=0}
$$

Since the straight segment precedes the fifth-order Bezier transition segment, the velocity must satisfy:

$$
\left\{\begin{array}{l}
\dot{u_{0}}=0 \\
\dot{u_{1}}=|\dot{b}(u)|_{u=0} \mid \cdot \lambda / l_{A B}
\end{array}\right.
$$

where $l_{A B}$ is the path function for straight segment; $\lambda$ is the scale factor of the fifth-order Bezier transition segment. To constraint the start and end of the arc segment, the continuous velocity and acceleration, straight segment displacement, acceleration, maximum velocity, maximum acceleration and maximum jerk must satisfy: $u_{0}=0, u_{1}=1, \ddot{u}_{0}=\ddot{u}_{1}=0, \dot{u}_{\max }=$ $v_{\max } / r_{B C}, \ddot{u}_{\max }=a_{\max } / r_{B C}$ and $\ddot{u}_{\max }=j_{\max } / r_{B C}$.

In the path, the arc segment is at the end segment. Then, the velocity must satisfy:

$$
\left\{\begin{array}{l}
\dot{u_{0}}=|\operatorname{dotb}(u)|_{u=1} \mid \cdot \lambda / r_{B C} \\
\dot{u_{1}}=0
\end{array}\right.
$$

where $r_{B C}$ is the path function for the arc segment.

\section{Experimental verification}

\subsection{ROS-based modelling of rehabilitation manipulator}

The URDF is a standard ROS XML format in the ROS for robot modelling [24]. The ROS also provides a $C++$ parser for URDF files. This parser helps to implement motion planning or model-based computing. The model-based computing is easy to complete, for the information of the model is directly called in the algorithm code [11].

After URDF modelling, the MoveIt! Setup Assistant was employed to configure the robot model. Firstly, the Semantic Robot Description Format (SRDF) file was generated based on the user-imported robot model, creating a feature pack for the MoveIt! configuration. Then, the robot was configured, visualized and simulated. The resulting configuration files were stored in the "config" folder. The SRDF file saves all the information of the visual configuration, the URDF file stores the compiled information, the "kinematic.yaml" file retains the configuration of the kinematic solver kdl_kinmatics_plugin. In the "launch" folder, a series of launch files were generated automatically for the launch of the manipulator. The move_group is the core of the MoveIt! architecture [13]. Both kinematics solution and motion planning need the launch file move_group to start the manipulator. The MoveIt! Setup Assistant offers various algorithms for robot motion planning. Each of these algorithms is a plugin with a unique focus.

MoveIt! System Configuration Frameworkas shown in the Figure 3, the move_group [10] integrates various functions to the MoveIt! environment through plugins. Thus, all external information, including their interactions with the internal data must be implemented by MoveIt!. In this paper, the Open Motion Planning Library (OMPL) in MoveIt! is adopted to realize the robot functions [19]. The OMPL, as an open-source library, contains various cutting-edge algorithms. It is used by MoveIt! to plan the robot motion from start to destination. 


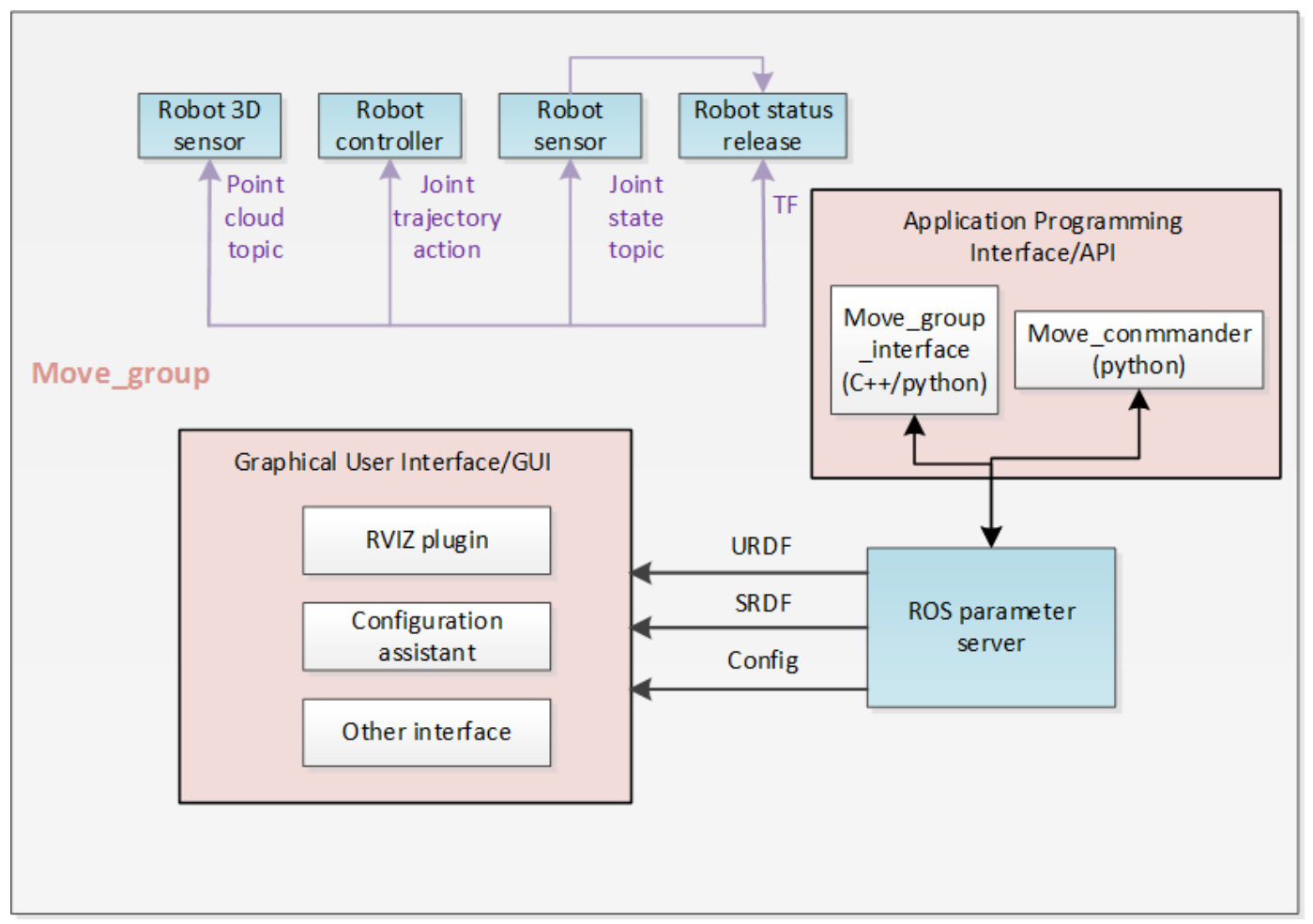

Figure 3: MoveIt! System Configuration Framework

\subsection{Experimental design based on impedance control}

In the ROS, the built-in rqt_plot provides the drawing functions. However, this function is too simple to visualize a huge amount of data. Hence, the Matlab was selected for data analysis. The whole operation flow chart as shown in the Figure 4. To avoid controlling the manipulator each time, the information about the topic joint_states was saved as a bag file. The command order as follows:

1). rosbag record -O manipulater1.bag /joint_states. "Create a trajectory motion simulation namedjoint_states. $\mathrm{O}$ is the indicator of the output file; manipulater1 is the name of the output file".

2). rosbagplay youbot_action2.bag -clock."A terminal was turned on to play back the data, clock is the simulation time".

3). rostopic echo/joint_states>record2.txt. "The output text was saved to store the data".

Finally, the text was copied to windows, imported to excel and analyzed via Matlab simulation. Figure 5 shows the modelled rehabilitation manipulator in virtual environment.

When the robot performs the auxiliary operation of basic rehabilitation movement, it is necessary to perform the basic rehabilitation actions such as the circular motion of the joint on a fixed track and the linear motion of push, pull and drag on the muscle or joint of the recovered person.

\subsection{Results analysis}

When the robot performs the basic auxiliary rehab operation, it is necessary to imitate people like action such as the joint circular motioning, and a fixed plan tracking,and the linear motion like pushing, pulling from muscle. Then, the first-order and second-order differential vectors of the trajectory should be computed, that is reached there habilitation exercise positions. 


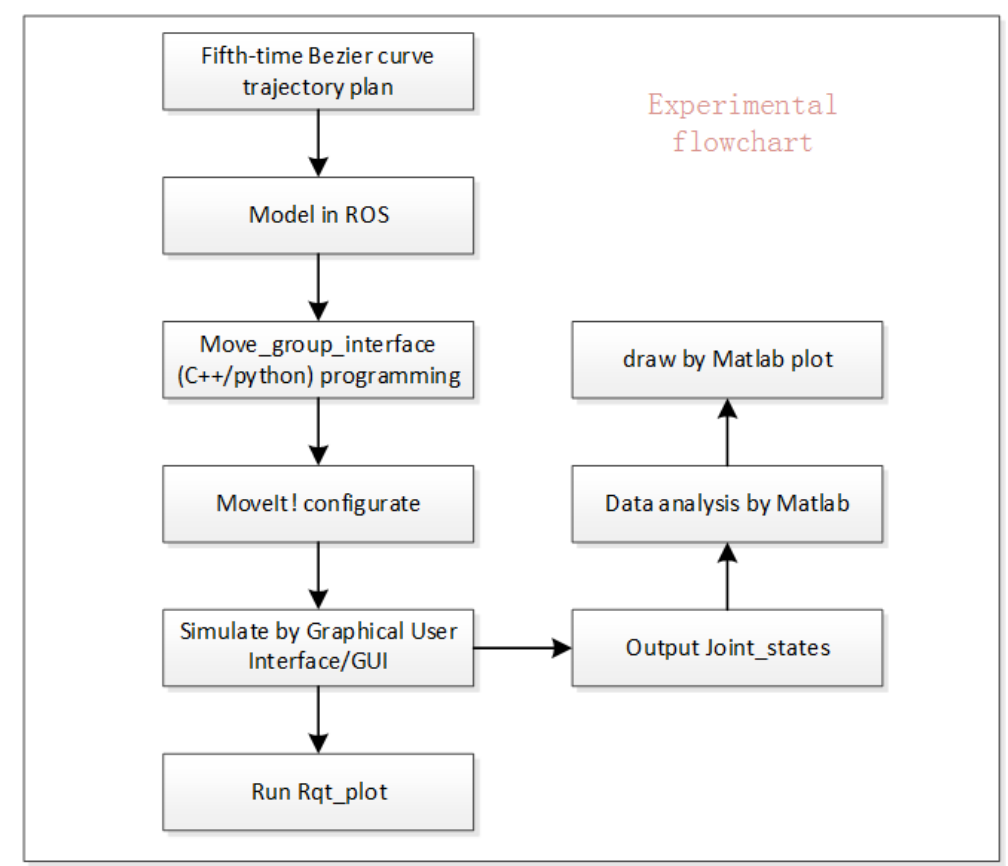

Figure 4: Experimental flowchart

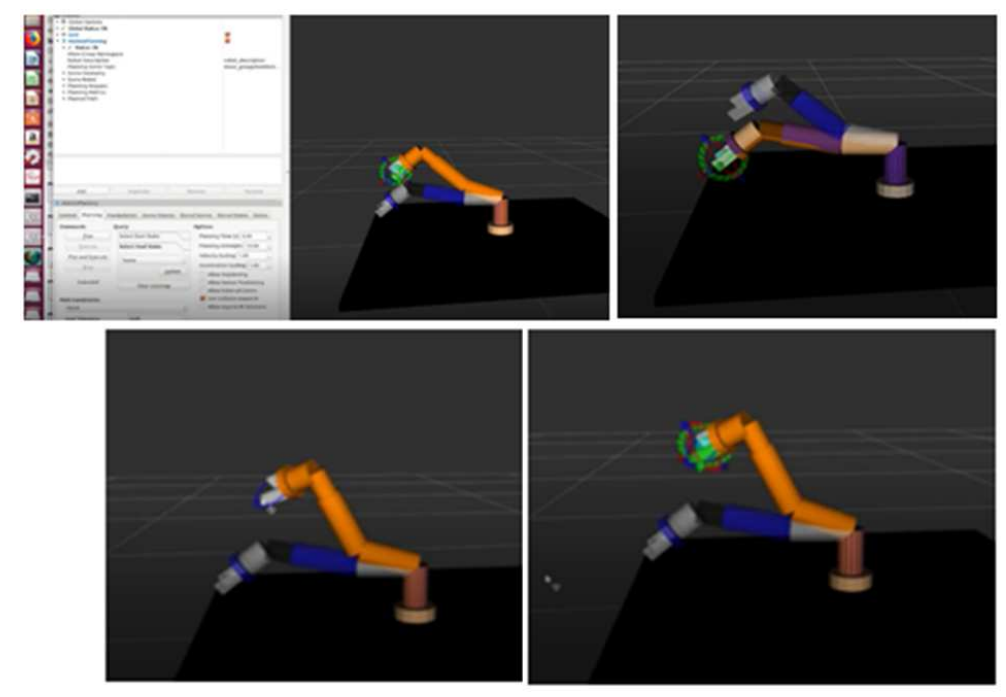

Figure 5: The Modelled Rehabilitation Manipulator 


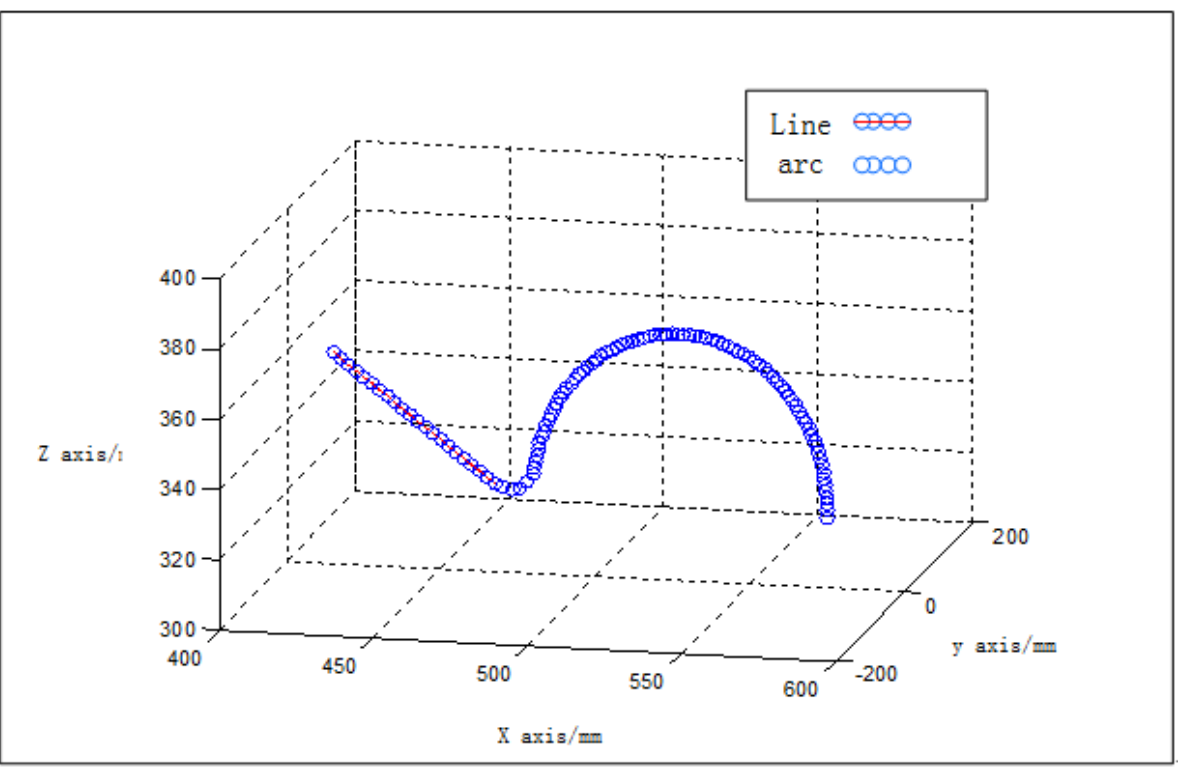

Figure 6: The Fifth-Time Bezier Curve of Rehabilitative Exercise

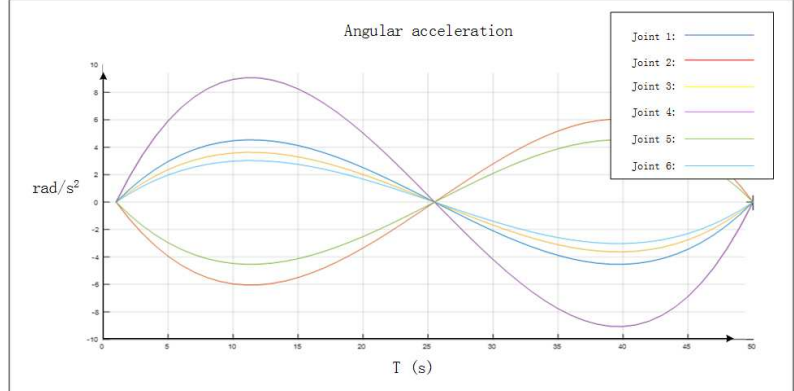

(a)

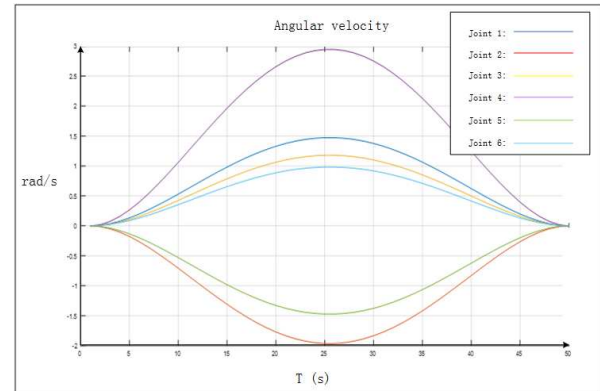

(b)

Figure 7: The Acceleration(a), Velocity (b) Variations of the End Effector in One Control Cycle
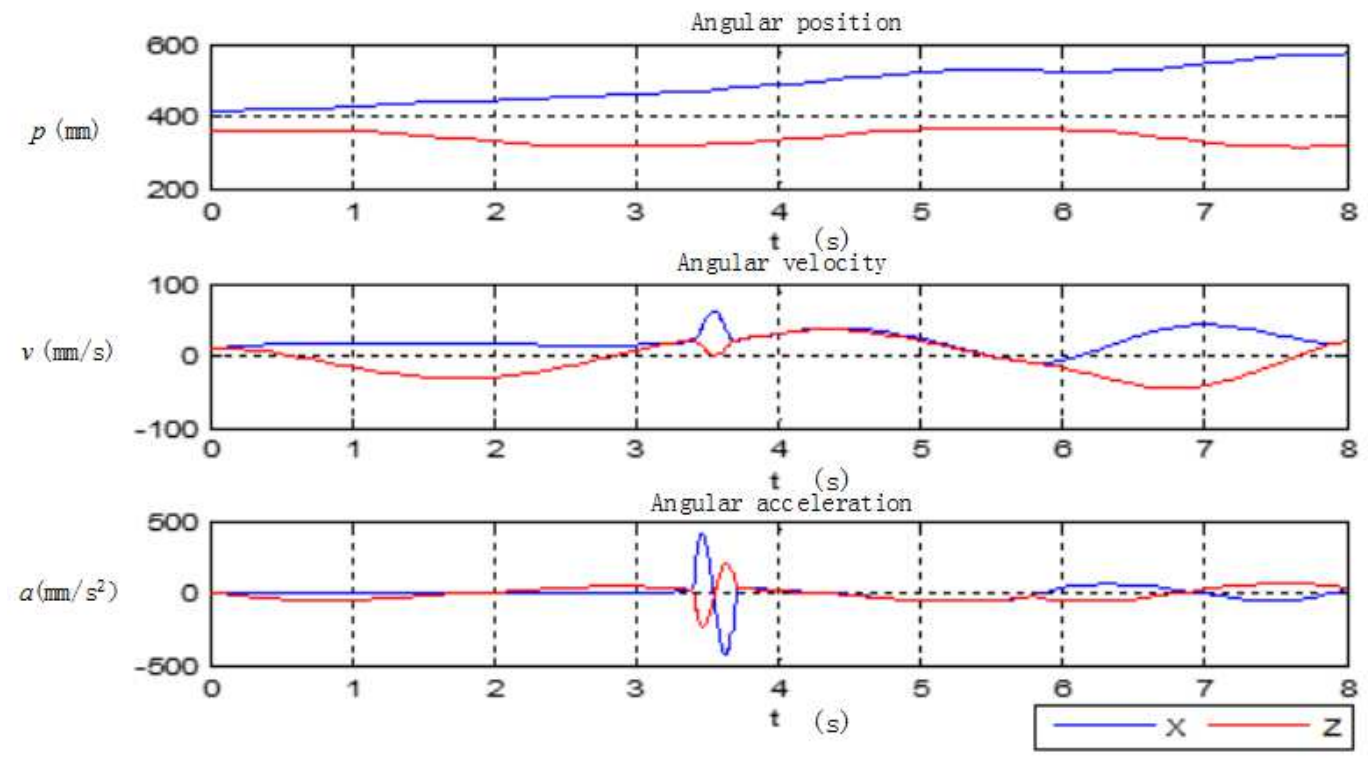

Figure 8: Position, Velocity and Acceleration of the End Effector 
The straight segment and arc segment was linked up by the fifth-time Bezier curve. We defined variable $\delta$ was $10 \mathrm{~mm}$.

The six points were calculated as the following information in the three-dimensional location:

$Q_{B_{0}}=(467.5932,0,325.3327), Q_{B_{1}}=(477.5448,0,324.2365)$,

$Q_{B_{2}}=(473.2109,0,323.7652), Q_{B_{4}}=(475.5001,0,324.5602)$,

$Q_{B_{5}}=(478.0034,0,325.8482)$ and $Q_{B_{6}}=(480.3521,0,328.0214)$.

The result of planning trajectory based on the fifth-time Bezier curve is shown in Figure 6.

The position, velocity and acceleration variations were plotted (Figure 7). It can be seen that the acceleration of the end effector changed smoothly, which is conducive to the rehabilitation efficiency and comfort.

Figure 8 presents the position, velocity and acceleration of end effector in the x-z plane. The acceleration curve at the end of the manipulator shows that our method slows down the abrupt changes in acceleration caused by rapid velocity change, when the end effector suddenly changes in the $\mathrm{x}-\mathrm{z}$ plane.

\section{Conclusions}

The hybrid control of position and force is essentially to select the DOF according to the desired path of the end effector, that is, to select the control component of the controller through $S$ and $\bar{S}$. In this paper, a force/position hybrid controller is designed based on the principle of impedance control. The control DOFs were selected through reconstruction of the desired path, and then the $S$ and $\bar{S}$ of the controller were identified. Next, our controller was tested in the ROS virtual simulation environment. The results show that our controller can facilitate and optimize the design of the path of rehabilitation robot.

\section{Acknowledgement}

This paper was supported by National sonar Key Laboratory Open Fund(6142109KF2018); National Fund Youth Project(11804263).

\section{Bibliography}

[1] Baksys, B.; Kilikevicius, S.; Chadarovicius, A. (2012). Experimental investigation of vibra assembly with passive compliance, Mechanika, 17(6), 608-611, 2012.

[2] Chang, J.; Wang, Y.Z.; Li, B. (2016). Accurate Operation Control Method Based on Hybrid Force/PositionAlgorithm for 7-DOF Manipulator, ROBOT, 38(5), 531-539, 2016.

[3] Hogan, N. (1985). Impedance control-An approach to manipulation. I - Theory. II - Implementation. III- Applications, Journal of Dynamic Systems Measurement \& Control, 107(1), $1-24,1985$.

[4] Hu, L.Y.; Zhan, J.M.(2015). Studyon theorthogonalization forHybrid motion/force control and its application in aspheric surface polishing,International Journal of Advanced Manufacturing Technology, 77(5-8), 1259-1268, 2015.

[5] Huang, H.; Gong, Z.M.; Chen,X.Q.; Zhou, L.(2002). Robotic grinding and polishing for turbine-vane overhaul, Journal of Materials Processing Technology, 127(2), 140-145, 2002. 
[6] Huang, T.; Sun, L.N.; Wang, Z.H.; Yu, X.Y.; Chen, G.D. (2017). Hybrid Force/Position Control Method for Robotic Polishing Based onPassive Compliance Structure, ROBOT, 39(6), 776-785 and 794, 2017.

[7] Kilikevicius, S.; Baksys, B. (2011). Dynamic analysis of vibratory insertion process, Assembly Automation, 31(3), 275-283, 2011.

[8] Li, Q.L.; Ye, T.M.(2009). Controlled assistance by an exoskeletal rehabilitation robot for upper limbs, Journal of Harbin Engineering University, 34(2), 166-177, 2009.

[9] Li, S.R.; Yin, H.Q.(2017). Adaptive position/force control for coordinated multiple flexiblejointmanipulators with time delay, Control Theory and Applications, 34(9), 1208-1214, 2017.

[10] Liu, Y.Q.; Tao, R.; Ding, F.; Zhang, J.X.; Chen, Y.J.; Liu, X.(2018). Research on vision servo 7DOF manipulator simulationbased on ROS and EtherCAT, Journal of natural science of Heilongjiang university, 35(1), 94-101, 2018.

[11] Lu, L.; Xie, S.X. (2018). Construction method of robot simulation model in ROS environment. Modern Electronics Technique, 41(7), 102-105, 110, 2018.

[12] Pan, L.Z.; Song, A.G.(2012). Real-time Safety Control of Upper-limb Rehabilitation Robot, ROBOT, 34(2), 197-210, 2012.

[13] Qi, Z.G.; Huang, P.F.; Liu, Z.X.; Han, D.(2019). Research on path planning method of spatial redundant manipulator. ActaAutomaticaSinica, 45(6), 1103-1110, 2019.

[14] Qin, H.Q.; Xiong, Q.Y.; Shi, X.(2014). Simulation research of force control system for manipulator based on Matlab, Computer Engineering and Applications, 50(12), 242-246, 2014 .

[15] Roy, N.; Newman, P.; Srinivasa, S. (2012). Rigidity maintenance control for multi-robot systems. Robotics: Science \& Systems VIII, MIT Press, 2012.

[16] Singh, H.P.; Sukavanam, N. (2013). Stability analysis of robust adaptive hybrid position/force controller for robot manipulators using neural network with uncertainties, Neural Computing \& Applications, 22(7-8), 1745-1755, 2013.

[17] Sun, Y.X.; Chen, W.D. (2011). Operating Unknown Constrained Mechanisms Based on Motion Prediction and Impedance Control, ROBOT, 33(5), 563-569, 2011.

[18] Wang, T. (2016). Design of An Exoskeleton Rehabilitation Robot Replicating the Synergistic Characteristics of Upper Extremity Movements, Huazhong University of Science and Technology, 2016.

[19] Wang, Z.G.; Freidovich, L.B.; Zhang, H.H.(2019). Periodic Motion Planning and Control for Double Rotary Pendulum via Virtual Holonomic Constraints,IEEE/CAA Journal of AutomaticaSinica, 6(1), 291-298, 2019.

[20] Wei, H.X.; Wang, X.D;Meng, Q.X.(2000). The study of a passive and pliant underwater claw, MarineTechnology,2000(3):17-20, 2000.

[21] Whitney, D.E.; Rourke, J.M.(1986). Mechanical behavior and design equations for elastomer shear pad remote center compliances, Journal of Dynamic Systems Measurement \& Control, 108(3), 223-232, 1986. 
[22] Xu, Q.S. (2013). Adaptive discrete-time sliding mode impedance control of a piezoelectric microgripper, Robotics IEEE Transactions on, 29(3), 663-673, 2013.

[23] Zhang, X.L.; Gu, X.X.; Zhao, H.F.; Wang, K.(2016). Design of a Compliant Robotic Arm Based on Series Elastic Actuator,ROBOT, 38(4), 385-394, 2016.

[24] Zuo, X.R.; Han, L.L.; Zhuang, J.; Shi, Q.Q.; Huang, Y.(2015). Design of human-robot interaction system for space robot using robot operating system, Computer engineering and design, 36(12), 3370-3374, 2015. 